Research Article

J Exp Clin Med

2021; 38(4): 583-588

doi: 10.52142 /omujecm. 38.4 .33

\title{
The role of triglyceride glucose index in predicting in-hospital adverse cardiovascular outcomes in patients with acute coronary syndrome
}

\author{
${ }^{1}$ Department of Cardiology, Servergazi State Hospital, Denizli, Turkey \\ ${ }^{2}$ Department of Cardiology, Faculty of Medicine, Pamukkale University, Denizli, Turkey
}

Sara Çetin ŞANLIALP ${ }^{1, *}$ (D), Gökay NAR ${ }^{2}$ (D)

\begin{tabular}{ccccc}
\hline Received: 10.04 .2021 & $\bullet$ & Accepted/Published Online: 04.05.2021 & Final Version: 30.08 .2021 \\
\hline
\end{tabular}

\begin{abstract}
Previous studies have shown the association of triglyceride glucose (TyG) index with metabolic syndrome (MetS), cardiovascular disease (CVD) and long-term adverse cardiovascular outcomes. However, to best our knowledge, the relation between the TyG index and in-hospital adverse cardiovascular outcomes in acute coronary syndrome (ACS) has not yet been reported. Hence, in this study, we aimed to evaluate the role of the TyG index in predicting in-hospital adverse cardiovascular outcomes in ACS and to compare its performance with the Global Acute Coronary Events Register (GRACE) risk score. 170 patients diagnosed with ACS and underwent coronary angiography were analyzed retrospectively. The TyG index was calculated using the following formula: $\ln$ [fasting triglycerides $(\mathrm{mg} / \mathrm{dL}) \times$ fasting blood glucose $(\mathrm{mg} / \mathrm{dL}) / 2$ ]. Receiver operating characteristics (ROC) curve analysis was used to evaluate the performance of the TyG index and GRACE risk score in predicting in-hospital adverse cardiovascular outcomes. A binary logistic regression model was applied to determine the independent predictors for in-hospital adverse cardiovascular outcomes. At the initial analysis, patients with adverse cardiovascular outcomes had higher TyG index and GRACE risk score $(\mathrm{p}=0.011, \mathrm{p}<0.001)$. In ROC curve analysis, the GRACE score performed better in predicting in-hospital adverse cardiovascular outcomes compared to TyG index (AUC:0.716, $\mathrm{p}<0.001$; AUC:0.588, $\mathrm{p}=0.054$ respectively). In binary logistic regression analysis, left ventricular ejection fraction (LVEF), multi-vessel disease and GRACE risk score were independent predictors for in-hospital adverse cardiovascular outcomes (OR: 0.840, 95\% CI: 0.791-0.891, p<0.001; OR: 3.581, 95\% CI:1.382-9.282, p=0.009; OR=1.017, 95\% CI: 1.001-1.034, p=0.04 respectively). Our study findings revealed that the TyG index was scant in predicting in-hospital adverse cardiovascular outcomes compared to GRACE risk score. The independent predictors for in-hospital adverse cardiovascular outcomes were LVEF, multivessel disease and GRACE risk score.
\end{abstract}

Keywords: acute coronary syndrome, cardiovascular outcomes, GRACE, triglyceride glucose index

\section{Introduction}

Cardiovascular disease (CVD) is still one of the leading causes of mortality and morbidity today. Despite favorable advances in treatment, the increase in dysmetabolic diseases such as hypertension, diabetes and hyperlipidemia cause a slower decrease in CVD-related deaths $(1,2)$. Insulin resistance (IR) associated with glycolipid disorders has become an important risk factor for CVD (3). In addition, there is constant evidence that IR may lead to atherosclerosis process and adverse cardiovascular events through inducing of proinflammatory cytokines, impairment of endothelial dysfunction, triggering of pro-coagulant factor expression and increased oxidative stress (4).

Recently, the triglyceride glucose (TyG) index derived from triglyceride and glucose has been preferred for IR evaluation due to not requiring special techniques and low cost (5). The studies have shown that TyG index may be associated with coronary artery calcification, arterial stiffness, carotid atherosclerosis, and coronary artery disease
(CAD) (6). In addition, some studies have revealed an association between the TyG index with adverse cardiovascular outcomes, both in the general population and in patient cohorts $(7,8)$. However, to the best of our knowledge, no study has been reported on the relation between the TyG index with in-hospital adverse cardiovascular outcomes and its comparison with The Global Registry of Acute Coronary Events (GRACE) risk score in acute coronary syndrome (ACS). Thus, in this study, we aimed to examine the relation between TyG index and inhospital adverse cardiovascular outcomes in ACS and to compare its performance with the GRACE risk score.

\section{Materials and methods}

\subsection{Study population}

In this retrospective observational study, 198 consecutive patients who were hospitalized for ACS and underwent coronary angiography at our tertiary care center between January 2020 and September 2020 were included. Malignancy, acute infection, severe liver failure, kidney and thyroid dysfunction, pregnancy, being under fibrate 
treatment and missing data were defined as exclusion criteria, and as a result of the final analysis, the study was conducted with a total of 170 patients.

This study was in compliance with the Helsinki Declaration of Human Rights and was approved by the local institutional ethical committee (Pamukkale University Faculty of Medicine Hospital, Denizli, Turkey; 22.12.2020/24, protocol no: 60116787-020). Informed consent was obtained from each patient before participating in the study.

\subsection{Data collection and definition}

Demographic and clinical data including age, gender, smoking, medical history, standard laboratory parameters and, angiographic images were analyzed retrospectively. The TyG index was calculated using the following formula: In [fasting TG (mg/dL) ×fasting blood glucose $(\mathrm{mg} / \mathrm{dL}) / 2$ ] (9). GRACE risk score consisting of age, systolic blood pressure, heart rate, presence of cardiac arrest, Killip class, ST segment deviation, serum creatinine, and positive cardiac markers was calculated for each patient using data from the registry system (10). ACS was defined as a collection of clinical syndromes, including unstable angina (UA), nonST- elevation myocardial infarction (STEMI), and STelevation myocardial infarction (STEMI). Hypertension was defined as blood pressure $\geq 140 / 90 \mathrm{mmHg}$ or currently taking antihypertensive treatment. Diabetes was defined as plasma glucose $\geq 200 \mathrm{mg} / \mathrm{dL}$ at any time or fasting blood glucose $\geq 126 \mathrm{mg} / \mathrm{dL}$ or under treatment. In-hospital adverse cardiovascular outcomes included cardiac death, cardiogenic shock, significant arrhythmia, recurrent revascularization and heart failure. The experience of any of these was defined as an in-hospital adverse cardiovascular outcome.

\subsection{Statistical analysis}

The data were analyzed using SPSS v.21.0 Windows (SPSS, Inc., Chicago, Ill., USA) programme package. Continuous variables were expressed as mean $\pm \mathrm{SD}$ or median, and categorical variables were presented as frequency and percentage. Kolmogorov-Smirnov test was used to determine the normal distribution and the comparisons based on normality distribution were done with Student's t-test or Mann-Whitney U test. Categorical variables were compared using $\chi 2$ test. Pearson's or Spearman correlation analysis was used to evaluate the relationship between the continuous variables. A binary logistic regression analysis was used to determine whether the TyG index was an independent predictor for in-hospital adverse cardiovascular outcomes. The performance of the TyG index and GRACE scores were compared using the receiver operating characteristics (ROC) curve analysis in predicting in-hospital adverse cardiovascular outcomes, and $\mathrm{p}<0.05$ was considered statistically significant.

\section{Results}

Patients without in-hospital adverse cardiovascular outcomes were assigned as group $1(\mathrm{n}=103)$ and those with in-hospital adverse cardiovascular outcomes were assigned as group 2 $(n=67)$. The demographic and clinical characteristics of the groups are presented in Table 1. There were no significant differences in mean age, male gender, smoking, hypertension, previous MI or revascularization history between the groups. However, diabetes incidence was significantly higher in group $2(\mathrm{p}=0.044) .27 \%, 47 \%, 26 \%$ of the patients were diagnosed with UA, NSTEMI and STEMI, respectively in group 1 . In group 2, 9\%, 31\% and 59\% of patients had UA, NSTEMI and STEMI respectively. While the percentages of single and two-vessel disease were higher in group 1, multi-vessel disease was more common in group 2. There were significant differences in LVEF, glucose, HbA1c, creatinine, WBC between the groups. However, lipid parameters, hemogram, TG/HDL-C, LDL-C/HDL-C were similar. When the groups were compared in terms of TyG index and GRACE risk score, TyG index and GRACE risk score of group 2 increased significantly $(9.00 \pm 0.70 \mathrm{vs}$ $9.30 \pm 0.82, \quad \mathrm{p}=0.011 ; \quad 114.42 \pm 25.68$ vs $138.76 \pm 33.16$, $\mathrm{p}<0.001$ respectively).

A comparison of in-hospital adverse cardiovascular outcomes based on the median TyG index of the study population is shown in Table 2. There were no significant differences in incidence of cardiogenic shock, heart failure, cardiac death and significant arrhythmia in patients with low and high TyG index. However, the incidence of recurrent ischemia increased in patients with high TyG index compared to those with low TyG index $(p=0.02)$. There was no difference between the groups in terms of single- or twovessel disease, however multi-vessel disease was more common in patients with high TyG index $(\mathrm{p}=0.042)$ (Table 3).

In correlation analysis, the TyG index showed a significant association with hypertension, diabetes, NSTEMI, LVEF, HbAlc, lipid parameters, creatinine, WBC, and multi-vessel disease (Table 4). However, the performance of the TyG index in predicting in-hospital adverse cardiovascular outcomes was not at expected level in the ROC curve analysis (95\% CI $=0.501-0.674$, AUC $=0.588$, 94\% sensitivity, 25\% specificity, $\mathrm{p}=0.054$ ). The GRACE risk score predicted in-hospital adverse cardiovascular outcomes with $58 \%$ sensitivity and $81 \%$ specificity at a cut-off value of 135.50 (95\% CI $=0.633$ $0.799, \mathrm{AUC}=0.716, \mathrm{p}<0.001)$ and its performance was better compared to the TyG index (Fig. 1). In binary logistic regression analysis, the parameters associated with inhospital adverse cardiovascular outcomes were LVEF, multi-vessel disease and GRACE risk score, regardless of all causes, as presented in Table 5 . 

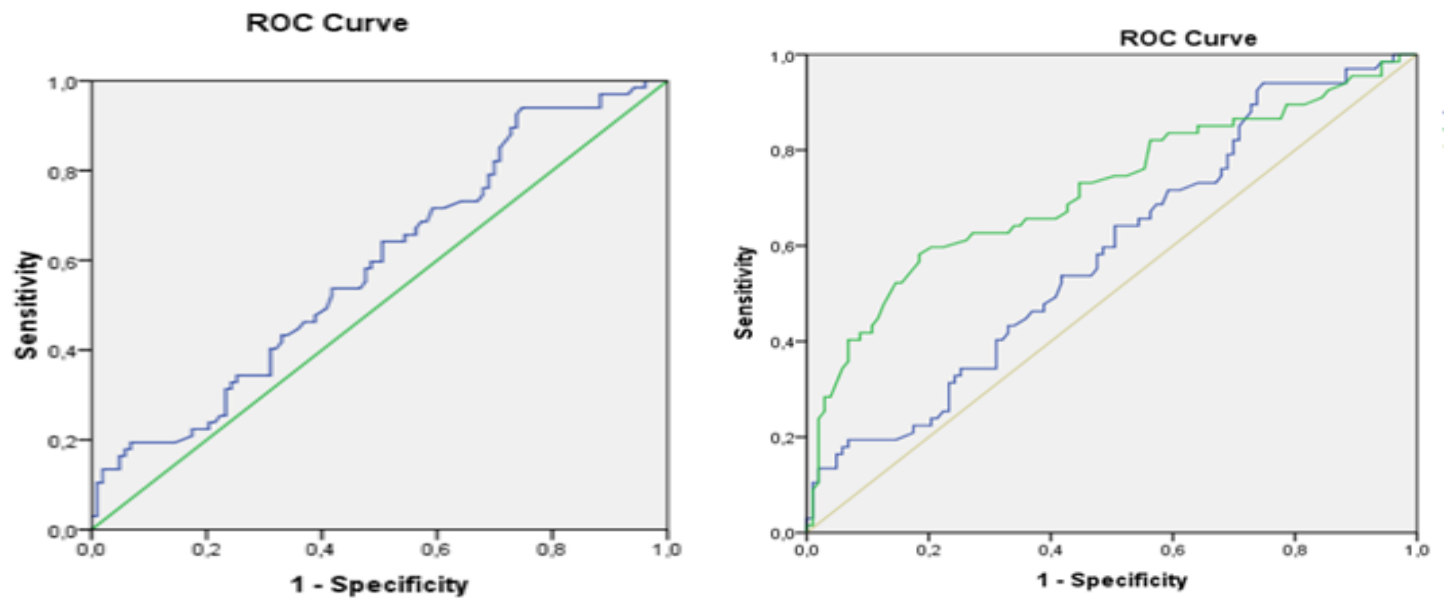

Fig. 1. Receiver operating characteristic (ROC) curves in predicting in-hospital adverse cardiovascular outcomes

Table 1. Baseline characteristics and clinical data of study population

\begin{tabular}{|c|c|c|c|}
\hline Variables & Group I ( $\mathrm{n}=103)$ & Group II (n=67) & p- value \\
\hline Mean age (years) & $65.01 \pm 11.28$ & $67.78 \pm 15.29$ & 0.177 \\
\hline Male gender, $\mathrm{n}(\%)$ & $65(63)$ & $40(60)$ & 0.412 \\
\hline Hypertension, $\mathrm{n}(\%)$ & $57(55)$ & $31(46)$ & 0.159 \\
\hline Diabetes, $\mathrm{n}(\%)$ & $33(32)$ & $31(46)$ & 0.044 \\
\hline Current smoking, n (\%) & $34(33)$ & $25(37)$ & 0.339 \\
\hline Previous MI, $\mathrm{n}(\%)$ & $9(9)$ & $7(10)$ & 0.709 \\
\hline PCI history, $\mathrm{n}(\%)$ & $6(6)$ & $4(6)$ & 0.969 \\
\hline CABG history, $\mathrm{n}(\%)$ & $7(7)$ & $6(9)$ & 0.605 \\
\hline UA, n (\%) & $28(27)$ & $6(9)$ & 0.004 \\
\hline NSTEMI, $\mathrm{n}(\%)$ & $48(47)$ & $21(31)$ & 0.034 \\
\hline STEMI, n (\%) & $27(26)$ & $40(59)$ & $<0.001$ \\
\hline LVEF \%, (median) & 55.00 & 35.00 & $<0.001$ \\
\hline Blood glucose, mg/dL (median) & 120.00 & 174.00 & $<0.001$ \\
\hline HbAlc $\%,($ median $)$ & 7.60 & 8.80 & 0.047 \\
\hline Creatinine, mg/dL (median) & 0.86 & 0.96 & 0.004 \\
\hline Tchol, $\mathrm{mg} / \mathrm{dL}$ & $173.99 \pm 39.19$ & $171.37 \pm 41.45$ & 0.678 \\
\hline LDL-C,mg/dL & $104.14 \pm 34.86$ & $106.12 \pm 34.70$ & 0.717 \\
\hline HDL-C, mg/dL (median) & 39.00 & 40.00 & 0.952 \\
\hline $\mathrm{TG}, \mathrm{mg} / \mathrm{dL}$ (median) & 118.00 & 130.00 & 0.275 \\
\hline Hemoglobin, g/dL (median) & 13.20 & 12.80 & 0.288 \\
\hline WBC, cells $/ \mu \mathrm{L}$ (median) & 9.08 & 10.70 & $<0.001$ \\
\hline TyG index & $9.00 \pm 0.70$ & $9.30 \pm 0.82$ & 0.011 \\
\hline LDL-C/HDL-C & $2.7 \pm 1.16$ & $2.8 \pm 1.19$ & 0.548 \\
\hline TG/HDL-C & 3.27 & 3.35 & 0.557 \\
\hline Single-vessel disease, $\mathrm{n}(\%)$ & $39(38)$ & $12(18)$ & 0.006 \\
\hline Two-vessel disease, $\mathrm{n}(\%)$ & $35(34)$ & $10(15)$ & 0.006 \\
\hline Multi-vessel disease, $\mathrm{n}(\%)$ & $29(28)$ & $45(67)$ & $<0.001$ \\
\hline GRACE risk score & $114.42 \pm 25.68$ & $138.76 \pm 33.16$ & $<0.001$ \\
\hline
\end{tabular}

MI, myocardial infarction; PCI, percutaneous coronary intervention; CABG, coronary artery bypass graft; UA, unstable angina; NSTEMI, non- ST- elevation myocardial infarction; STEMI, ST- elevation myocardial infarction; LVEF, left ventricular ejection fraction; TChol; total cholesterol; LDL-C, low-density lipoprotein cholesterol; HDL-C, high-density lipoprotein cholesterol; TG, triglycerides; WBC, white blood cells; TyG index, triglyceride glucose index

Table 2. The comparison of in-hospital adverse cardiovascular outcomes between the groups based on median TyG index

\begin{tabular}{|c|c|c|c|}
\hline Variables & Low $(<9.04)(\mathrm{n}=84)$ & High $(>9.04)(\mathrm{n}=86)$ & $\mathrm{p}$ \\
\hline Cardiogenic shock & - & $2(2)$ & 0.160 \\
\hline Heart failure & $29(35)$ & $32(37)$ & 0.715 \\
\hline Significant arrhythmia & $7(8)$ & $14(16)$ & 0.115 \\
\hline Recurrent ischemia & - & $9(11)$ & 0.002 \\
\hline Cardiac death & $4(5)$ & $11(13)$ & 0.065
\end{tabular}

TyG index, triglyceride glucose index 
Table 3. The number of diseased vessels according to median TyG index

\begin{tabular}{|l|c|c|c|}
\hline \multicolumn{1}{|c|}{ Variables } & $\begin{array}{c}\text { Low }(<9.04) \\
\mathrm{n}=84\end{array}$ & $\begin{array}{c}\text { High }(>9.04) \\
\mathrm{n}=86\end{array}$ & $\mathrm{p}$ \\
\hline $\begin{array}{l}\text { Single-vessel } \\
\text { disease, } \mathrm{n}(\%)\end{array}$ & $26(31)$ & $25(29)$ & 0.789 \\
\hline $\begin{array}{l}\text { Two-vessel } \\
\text { disease, } \mathrm{n}(\%)\end{array}$ & $28(33)$ & $17(20)$ & 0.154 \\
\hline $\begin{array}{l}\text { Multi-vessel } \\
\text { disease, } \mathrm{n}(\%)\end{array}$ & $30(36)$ & $44(51)$ & 0.042 \\
\hline
\end{tabular}

TyG index, triglyceride glucose index

Table 4. The correlation analysis of TyG index

\begin{tabular}{|l|c|c|}
\hline Variables & $\mathrm{r}$ & $\mathrm{p}$ \\
\hline Age & -0.105 & 0.173 \\
\hline Hipertension & 0.201 & 0.008 \\
\hline Diabetes & 0.477 & $<0.001$ \\
\hline NSTEMI & 0.292 & 0.012 \\
\hline LVEF & -0.217 & 0.005 \\
\hline HbA1c & 0.516 & $<0.001$ \\
\hline Creatinine & 0.228 & 0.003 \\
\hline Tchol & 0.248 & $<0.001$ \\
\hline LDL-C & 0.189 & 0.013 \\
\hline HDL-C & -0.300 & $<0.001$ \\
\hline WBC & 0.282 & 0.018 \\
\hline Multi-vessel disease & 0.346 & 0.027 \\
\hline GRACE risk score & 0.046 & 0.549 \\
\hline
\end{tabular}

NSTEMI, non- ST- elevation myocardial infarction; LVEF, left ventricular ejection fraction; TChol; total cholesterol; LDL-C, lowdensity lipoprotein cholesterol; HDL-C, high-density lipoprotein cholesterol; WBC, white blood cells; TyG index, triglyceride glucose index

Table 5. Binary logistic regression analysis for in-hospital adverse cardiovascular outcomes

\begin{tabular}{|l|c|c|c|c|}
\hline \multirow{2}{*}{ Variables } & \multirow{2}{*}{ OR } & \multicolumn{2}{|c|}{$95 \%$ CI } & \multirow{2}{*}{$\mathrm{p}$} \\
\cline { 3 - 4 } & & $\begin{array}{l}\text { Lower } \\
\text { bound }\end{array}$ & $\begin{array}{l}\text { Upper } \\
\text { bound }\end{array}$ & \\
\hline LVEF & 0.840 & 0.791 & 0.891 & $<0.001$ \\
\hline WBC & 1.098 & 0.968 & 1.246 & 0.146 \\
\hline $\begin{array}{l}\text { Multi-vessel } \\
\text { disease }\end{array}$ & 3.581 & 1.382 & 9.282 & 0.009 \\
\hline $\begin{array}{l}\text { GRACE risk } \\
\text { score }\end{array}$ & 1.017 & 1.001 & 1.034 & 0.040 \\
\hline TyG index & 1.158 & 0.602 & 2.227 & 0.660 \\
\hline
\end{tabular}

LVEF, left ventricular ejection fraction; WBC, white blood cells; TyG index, triglyceride glucose index

\section{Discussion}

In the current study, we investigated the impact of TyG index on in-hospital adverse cardiovascular outcomes in patients diagnosed with ACS at the first time and our main findings were as follows: (1) TyG index and GRACE risk score were higher in patients with in-hospital adverse cardiovascular outcomes; (2) There were no significant differences in inhospital adverse outcomes including heart failure, cardiac death, cardiogenic shock, and heart failure between patients with high and low TyG index. However, recurrent ischemia was more common in patients with high TyG index. In addition, TyG index was significantly correlated with dysmetabolic conditions such as hypertension, diabetes, dyslipidemia, and multi-vessel disease; (3) GRACE risk score performed better in predicting in-hospital adverse outcomes compared to TyG index; (4) LVEF, multivessel disease and GRACE risk score were independent predictors for in-hospital adverse cardiovascular outcomes.

Many studies have shown that IR is associated with CVD and cardiovascular outcomes, in both short-term and longterm prognosis (11). However, the pathophysiological mechanisms by which IR plays a role in CVD have not been clearly determined. Inflammation, oxidative stress, lipid metabolism disorders, disruption of endothelial dysfunction through decreased NO release and inducing of the coagulation cascade are blamed mechanisms (4).

TyG index, a new method for evaluating IR, is associated with dysmetabolic conditions and CVD. Recently, the data reported that patients with increased TyG index have a higher risk of hypertension and diabetes. Moreover, the studies have found that the subclinical CAD may be more prevalent during screening with coronary CT angiography in patients with a high TyG index (12-14). Similar to these studies, there was a significant association between TyG index with hypertension, diabetes and impaired lipid parameters in our study. We also found a significant correlation between multi-vessel disease with TyG index and Mao et al.'s study supported our study by showing an increased incidence of multi-vessel disease in NSTEMI patients with a high TyG index (8). Recently, the relationship of the TyG index with cardiovascular outcomes has been investigated and in one study conducted with stable CAD patients; a high TyG index was associated with primary endpoints including all-cause death, non-fatal MI, recurrent revascularization and stroke (7). In another study, the TyG index showed successful performance in predicting cardiovascular events in patients with ACS, regardless of all causes (15). Additionally, the increased TyG index indicated the adverse cardiovascular outcomes in diabetic patients diagnosed with ACS undergoing PCI in one study (16). In this study, to the best our knowledge, we investigated the role of the TyG index on in- hospital adverse outcomes in ACS patients at the first time. Patients with in-hospital adverse cardiovascular outcomes at baseline showed a higher TyG index. However, the TyG index failed to predict adverse in-hospital cardiovascular outcomes compared to the GRACE risk score. Also, TyG index was not an independent predictor for in-hospital adverse cardiovascular outcomes after adjusting for confounding factors. In all above studies, it was aimed to determine the long-term prognostic significance of the TyG index in CAD patients, not inhospital adverse cardiovascular outcomes. However, adverse cardiovascular events observed during hospitalization after ACS were reported in our study. in-hospital adverse 
cardiovascular outcomes may be more affected by hemodynamic status at admission, late hospitalization due to atypical angina, presence of previous $\mathrm{CAD}$, late referrals from rural areas, and inclusion of patients with unsuccessful revascularization from an external center, rather than TyG index. However, the TyG index was significantly correlated with in-hospital recurrent ischemia in our study. This may be due to the TyG index's association with dysmetabolic conditions such as hypertension, diabetes, hyperglycemia, and lipid metabolism disorders, which predispose to atherosclerosis.

Another finding of our study was that the GRACE risk score, a clinical scoring, performed better in predicting inhospital adverse cardiovascular outcomes compared to the TyG index and was an independent predictor for in-hospital adverse cardiovascular outcomes. Indeed, clinical evaluation may be better than laboratory parameters in predicting inhospital adverse cardiovascular outcomes that may occur immediately after ACS. As a matter of fact, the GRACE risk score, developed from multinational prospective patient registries, has been accepted as a strong predictor of shortterm prognosis in patients with ACS, and its use has been recommended by ESC guidelines. (17). The other independent predictors for in-hospital adverse cardiovascular outcomes were LVEF and multi-vessel disease in our study. In a study with 8983 ACS patients, LVEF at admission was an independent predictor of death and adverse cardiovascular outcomes (18). Also, low LVEF may have led to clinical instability in patients with in-hospital adverse cardiovascular outcomes in our study. In another study, multivessel disease was a more important predictor of in-hospital adverse cardiovascular outcomes in patients with ACS compared to TIMI and age (19), and the findings of this study were consistent with our study.

Our study had some limitations. First, our study was retrospective and the study sample was relatively small. Second, the study was conducted in a Turkish population, and the study findings may vary by ethnicity. Third, patients using antidiabetic agents were not excluded. Therefore, we cannot ignore the effects of antidiabetic drugs. Fourth, due to retrospective design, we had missing data such as body mass index, exercise status, dietary habits, and energy intake, which could affect patients' TyG index.

As a result, the TyG index was higher in patients with inhospital adverse cardiovascular outcomes. The performance of the GRACE risk score in predicting in-hospital adverse cardiovascular outcomes was better compared to the TyG index. Thus, the TyG index may not be a useful marker to predict in-hospital prognosis in patients diagnosed with ACS. According to the findings of our study, the independent predictors of in-hospital adverse cardiovascular outcomes were LVEF, multivessel disease, and GRACE risk score. However, a larger sample size, longer follow-up time, and multicenter studies are needed to confirm our findings.

\section{Acknowledgement}

There are no any acknowledgements

\section{Conflict of Interest}

The authors declare that there are no conflicts of interest.

\section{References}

1. Townsend N, Nichols $M$, Scarborough $P$, Rayner $M$. Cardiovascular disease in Europe--epidemiological update 2015. Eur Heart J. 2015 Oct 21;36(40):2696-705. doi: 10.1093/eurheartj/ehv428. Epub 2015. PMID: 26306399.

2. Sidney S, Quesenberry CP Jr, Jaffe MG, Sorel M, NguyenHuynh MN, Kushi LH, et al. Recent Trends in Cardiovascular Mortality in the United States and Public Health Goals. JAMA Cardiol. 2016;1(5):594-9. doi: 10.1001/jamacardio.2016.1326. PMID: 27438477.

3. Thai PV, Tien HA, Van Minh H, Valensi P. Triglyceride glucose index for the detection of asymptomatic coronary artery stenosis in patients with type 2 diabetes. Cardiovasc Diabetol. 2020;19(1):137. doi: 10.1186/s12933-020-01108-2. PMID: 32919465; PMCID: PMC7488689.

4. Ormazabal V, Nair S, Elfeky O, Aguayo C, Salomon C, Zuñiga FA. Association between insulin resistance and the development of cardiovascular disease. Cardiovasc Diabetol. 2018;17(1):122. doi: 10.1186/s12933-018-0762-4. PMID: 30170598; PMCID: PMC6119242.

5. Vasques AC, Novaes FS, de Oliveira Mda S, Souza JR, Yamanaka A, Pareja JC, et al. TyG index performs better than HOMA in a Brazilian population: a hyperglycemic clamp validated study. Diabetes Res Clin Pract. 2011;93(3):98-100. doi: 10.1016/j.diabres.2011.05.030. Epub 2011 Jun 12. PMID: 21665314.

6. Zhang Y, Ding X, Hua B, Liu Q, Gao H, Chen H, et al. High triglyceride-glucose index is associated with adverse cardiovascular outcomes in patients with acute myocardial infarction. Nutr Metab Cardiovasc Dis. 2020 ;30(12):23512362. doi: 10.1016/j.numecd.2020.07.041. Epub 2020 Aug 2. PMID: 32917496.

7. Jin JL, Cao YX, Wu LG, You XD, Guo YL, Wu NQ, et al. Triglyceride glucose index for predicting cardiovascular outcomes in patients with coronary artery disease. J Thorac Dis. $2018 ; 10(11): 6137-6146$. doi: 10.21037/jtd.2018.10.79. PMID: 30622785; PMCID: PMC6297409.

8. Mao Q, Zhou D, Li Y, Wang Y, Xu SC, Zhao XH. The Triglyceride-Glucose Index Predicts Coronary Artery Disease Severity and Cardiovascular Outcomes in Patients with NonST-Segment Elevation Acute Coronary Syndrome. Dis Markers. 2019;2019:6891537. doi: 10.1155/2019/6891537. PMID: 31281548; PMCID: PMC6594265.

9. Guerrero-Romero F, Simental-Mendía LE, González-Ortiz M, Martínez-Abundis E, Ramos-Zavala MG, Hernández-González $\mathrm{SO}$, et al. The product of triglycerides and glucose, a simple measure of insulin sensitivity. Comparison with the euglycemic-hyperinsulinemic clamp. J Clin Endocrinol Metab. 2010 Jul;95(7):3347-51. doi: 10.1210/jc.2010-0288. PMID: 20484475.

10. Fox KA, Dabbous OH, Goldberg RJ, Pieper KS, Eagle KA, Van de Werf F, et al. Prediction of risk of death and myocardial infarction in the six months after presentation with acute coronary syndrome: prospective multinational observational study (GRACE). BMJ. 2006;333(7578):1091. PMID:17032691; PMCID: PMC1661748. 
11. Laakso M, Kuusisto J. Insulin resistance and hyperglycaemia in cardiovascular disease development. Nat Rev Endocrinol. 2014 ;10(5):293-302. doi: 10.1038/nrendo.2014.29. Epub 2014 Mar 25. PMID: 24663222.

12. Sánchez-Íñigo L, Navarro-González $\mathrm{D}$, Pastrana-Delgado J, Fernández-Montero A, Martínez JA. Association of triglycerides and new lipid markers with the incidence of hypertension in a Spanish cohort. J Hypertens. 2016 ;34(7):1257-65. doi: 10.1097/HJH.0000000000000941. PMID: 27136314 .

13. Lee DY, Lee ES, Kim JH, Park SE, Park CY, Oh KW, et al. Predictive Value of Triglyceride Glucose Index for the Risk of Incident Diabetes: A 4-Year Retrospective Longitudinal Study. PLoS One. 2016;11(9):e0163465. doi: 10.1371/journal.pone.0163465. PMID: 27682598; PMCID: PMC5040250.

14. Park GM, Cho YR, Won KB, Yang YJ, Park S, Ann SH, et al. Triglyceride glucose index is a useful marker for predicting subclinical coronary artery disease in the absence of traditional risk factors. Lipids Health Dis. 2020;19(1):7. doi: 10.1186/s12944-020-1187-0. PMID: 31937313; PMCID: PMC6961240.

15. Hu C, Zhang J, Liu J, Liu Y, Gao A, Zhu Y, et al. Discordance between the triglyceride glucose index and fasting plasma glucose or $\mathrm{HbA} 1 \mathrm{C}$ in patients with acute coronary syndrome undergoing percutaneous coronary intervention predicts cardiovascular events: a cohort study from China. Cardiovasc Diabetol. 23;19(1):116. doi: 10.1186/s12933-020-01091-8. PMID: 32703284; PMCID: PMC7379768.
16. Ma X, Dong L, Shao Q, Cheng $\mathrm{Y}, \mathrm{Lv} \mathrm{S}$, Sun $\mathrm{Y}$, et al. Triglyceride glucose index for predicting cardiovascular outcomes after percutaneous coronary intervention in patients with type 2 diabetes mellitus and acute coronary syndrome. Cardiovasc Diabetol. 2020;19(1):31. doi: 10.1186/s12933-02001006-7. PMID: 32156279; PMCID: PMC7063826.

17. Hamm CW, Bassand JP, Agewall S, Bax J, Boersma E, Bueno $\mathrm{H}$, et al.; ESC Committee for Practice Guidelines. ESC Guidelines for the management of acute coronary syndromes in patients presenting without persistent ST-segment elevation: The Task Force for the management of acute coronary syndromes (ACS) in patients presenting without persistent STsegment elevation of the European Society of Cardiology (ESC). Eur Heart J. 2011 Dec;32(23):2999-3054. doi: 10.1093/eurheartj/ehr236. Epub 2011 . PMID: 21873419.

18. Perelshtein Brezinov O, Klempfner R, Zekry SB, Goldenberg I, Kuperstein R. Prognostic value of ejection fraction in patients admitted with acute coronary syndrome: A real world study. Medicine (Baltimore). 2017;96(9):e6226. doi: 10.1097/MD.0000000000006226. PMID: 28248882; PMCID: PMC5340455.

19. Muller DW, Topol EJ, Ellis SG, Sigmon KN, Lee K, Califf RM. Multivessel coronary artery disease: a key predictor of short-term prognosis after reperfusion therapy for acute myocardial infarction. Thrombolysis and Angioplasty in Myocardial Infarction (TAMI) Study Group. Am Heart J. 1991;121(4 Pt 1):1042-9. doi: 10.1016/0002-8703(91)90661-z. PMID: 1901190. 